\title{
The risks of
}

\section{heavy-hamded}

\section{broushing}

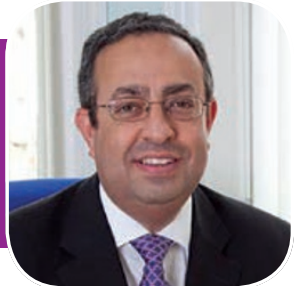

Professor Andrew Eder' considers the damage that can be caused to the teeth if patients are too heavy-handed with their toothbrushing, as well as exploring other potential causes of abrasion and offering preventive advice to be passed on to patients.

'Professor Andrew Eder is a Specialist in Restorative Dentistry and Prosthodontics and Clinical Director of the London Tooth Wear Centre, a specialist referral practice in Central London. He is also Professor/Honorary Consultant at the UCL Eastman Dental Institute and ProVice-Provost and Director of Life Learning at UCL.

The London Tooth Wear Centre offers an evidence-based and comprehensive approach to managing tooth wear, using the latest clinical techniques and a holistic approach in a professional and friendly environment.

www.toothwear.co.uk

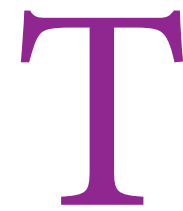

he Oxford Dictionary of Dentistry defines 'abrasion' as: 'The non-bacterial loss of tooth tissue due to frictional tooth wear by extrinsic

agents. Common causes are toothbrushing, particularly with abrasive pastes, pipe smoking, and pencil chewing. The lesions produced by toothbrush abrasion are typically wedge-shaped and are most commonly associated with the labial and buccal surfaces of the premolars, canines, and incisors of the permanent dentition'.

These are not the only cause of abrasion you can add to the list a diet rough in texture or using the teeth for a purpose other than nature intended, such as biting tags off new purchases, or cleaning between the teeth with tools not created for that purpose, for instance earring posts, keys and credit cards!

A patient suffering from tooth wear may report sensitivity, as well as problems chewing. Their teeth may also look shorter on smiling or when speaking. In addition:

- Teeth may become less white as some of the outer surface is lost

- Front teeth may become sharp or chipped

- Chewing surfaces may wear flat and take on a shiny, pitted appearance

- Restorations such as crowns and bridges may stand proud of the natural teeth.

Patient awareness and education Raising awareness and educating our patients 
about the potential for abrasive tooth wear is essential if we are to prevent further damage. Thus, for example, an important message to share is the importance of gentle but effective brushing - in my experience, many people mistake brushing hard for brushing well! It may be appropriate for the dental professional to demonstrate the best technique for the patient, and to recommend the use of a soft toothbrush and non-abrasive toothpaste.

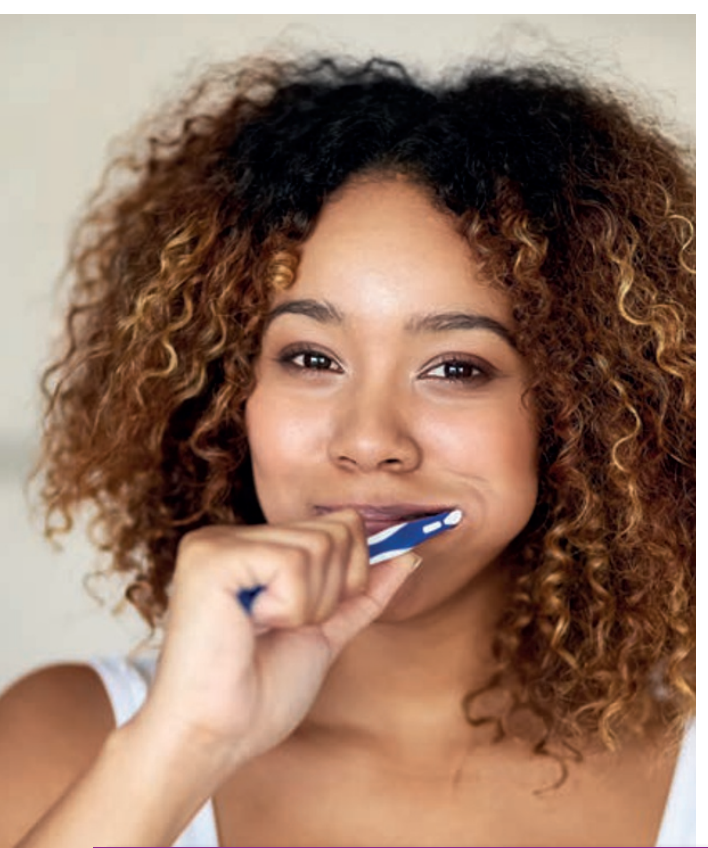

\section{'SOME OF THE MORE COMMONLY CONSUMED}

\section{FOODS THAT CAN CONTRIBUTE INCLUDE}

\section{CELERY, CARROTS, BROCCOLI, SEEDS AND NUTS...'}

Meanwhile, as already mentioned, foods with a rough texture will make matters worse, so it is worth having a discussion with patients about their diet. As a rule of thumb to share with them, if it's tough to chew and/ or fibrous, it may well be abrasive. Some of the more commonly consumed foods that can contribute to abrasion include celery, carrots, broccoli, apples, seeds and nuts.

It is also very important to make sure patients understand their teeth are not a handy tool, for example to tear labels off newly bought items or to rip open packets

祍 of sweets when their hands fail them! In addition, many people chew foreign objects such as pens and pencils, very often without realising. If a discussion with your patient brings such an issue to the fore, it might be worth suggesting they coat their chew-item of choice with a bitter-tasting solution designed for nail biters.
The reality of wear

As Wiegand and Schlueterwrote: 'Although toothbrushing is considered a prerequisite for maintaining good oral health, it also has the pential to have an impact on tooth wear, mith regard to dental erosion. number of factors, including not only the physical properties of the toothpaste and toothbrush used but also patient-related factors such as toothbrushing frequency and force of brushing. While abrasion resulting from routine oral hygiene can be considered as physiological wear over time, intensive ming further harm eroded surfaces surface layer.'.

Added to this, there is no doubt that tooth wear is on the increase: over three-quarters of adults show signs of tooth wear. Comparing most recent Adult Dental Health Survey with its predecessor, figures suggest wear in England has increased by $10 \%{ }^{2}$

Our awareness of these issues - combined with proactive patient care - is key to helping the UK population achieve dental longevity, as well as keeping them pain-free. In addition, while this article has focused on abrasion, it

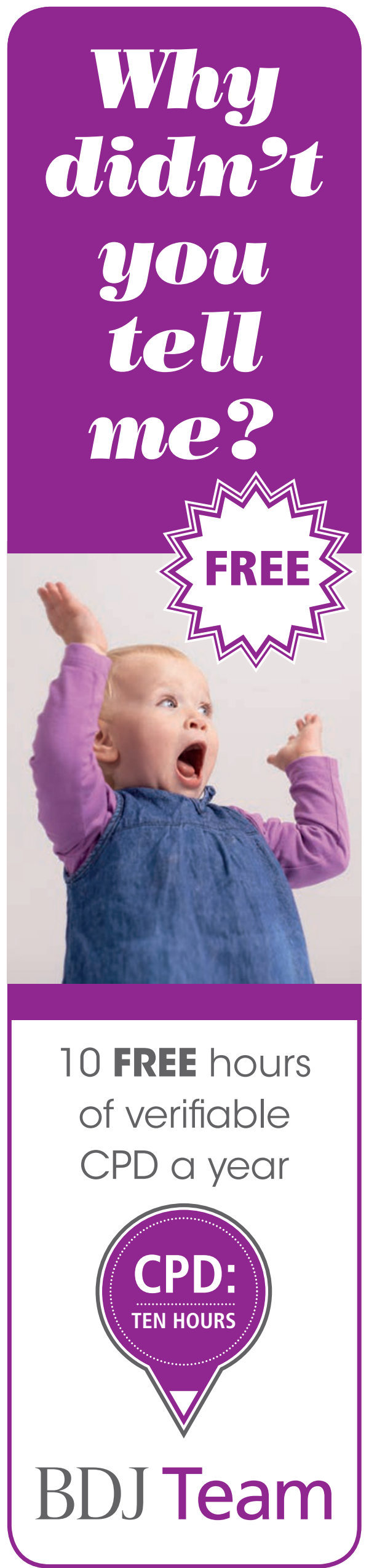

is important to note that tooth wear is multifactorial and should not be considered in isolation - abrasion, erosion, attrition and abfraction are rarely seen in isolation (if ever), and may affect people from all walks of life at any age.

The stark truth is that if patients remain unaware and uneducated about the potential for tooth damage through tooth wear, patients will continue with their destructive habits, which will have serious implications for their oral health in years to come.

1. Wiegand A, Schlueter N. The role of oral hygiene: does toothbrushing harm? Monogr Oral Sci 2014; 25: 215-219.

2. Adult Dental Health Survey 2009. Report 2: Disease and related disorders. Health and Social Care Information Centre, 2011.

bdjteam2017134 